\title{
Flexible Optical and Millimeter-Wave Analog-RoF Transmission with a Silicon-based Integrated Dual Laser Module
}

\author{
Devika Dass $^{(1)}$, Amol Delmade(1) $^{(1)}$ Liam Barry $^{(1)}$, Chris GH Roeloffzen ${ }^{(2)}$, \\ Douwe Geuzebroek ${ }^{(2)}$ and Colm Browning ${ }^{(1)}$
}

\author{
(1) School of Electronic Engineering, Dublin City University, Glasnevin, Dublin 9, D09 V209, Ireland. \\ devika.dass2@mail.dcu.ie \\ (2) LioniX International BV, 7521 AN Enschede, The Netherlands.
}

\begin{abstract}
A$ hybrid integrated $\ln P-S_{3} N_{4}$ dual tunable laser module is deployed as a highly flexible source for converged optical/mm-wave fronthaul. Experimental results show the wavelength flexible delivery of $5 \mathrm{G}$ signals over analog radio-over-fiber, incorporating wireless transmission at $60 \mathrm{GHz}$, with received EVMs as low as 5\%.
\end{abstract}

\section{Introduction}

The recent inclusion of millimeter-wave (mmwave) frequencies in $5 \mathrm{G}$ specifications has paved the way for the widespread commercialization and deployment of mobile communications systems initially utilizing carrier frequencies between 25 and $39 \mathrm{GHz}$. The enormous bandwidth demands envisioned for future applications such as autonomous vehicles (AV) and virtual reality (VR), coupled with the continued proliferation of small cell antenna sites, means that the expansion to higher mm-wave, and even terahertz ( $\mathrm{THz})$, frequencies is inevitable as we transition to the next generation of mobile communications ${ }^{[1]}$.

For many years, photonic solutions have been recognised as an effective means to generate electronic carriers at such high frequencies. More recently, research has focused on how these methods may be integrated with existing optical infrastructure (e.g. optical access networks) for $\mathrm{mm}$-wave generation and distribution - a concept known as 'convergence'[2,3]. Indeed, our previous works have explored how a pair of optical carriers with a mm-wave frequency difference may be distributed over an analog radio-over-fiber (ARoF) fronthaul link from a central office (CO) to a remote radio head $(\mathrm{RRH})$ for heterodyne detection; directly producing a mm-wave mobile signal after the photo-detection stage ${ }^{[4,5]}$. Although the A-RoF (i.e. multi-carrier modulation) transmission approach introduces stringent linearity, phase noise and frequency offset requirements ${ }^{[4]}$, it holds a distinct advantage over current digital (i.e single carrier) approaches in terms of both spectral efficiency and the potential for network scaling ${ }^{[6]}$.

Increasingly, flexible optical technologies such as tunable lasers, optical switch fabrics and active remote nodes are being proposed as a means to incorporate high bandwidth and low latency wavelength division multiplexing (WDM) networking in the access domain ${ }^{[7-10]}$. From the perspective of a converged network operating in such a dynamic environment, the ability to assign pairs of optical carriers in a flexible manner across a wide wavelength range for remote $\mathrm{mm}$ wave carrier generation would be highly advantageous.

A discrete approach to optical heterodyning involves the use of two independent tunable lasers whose wavelengths are separated by the desired mm-wave frequency. This method can provide a high level of tunability - but such operation typically requires the use of bulky and expensive sources as well as phase locking. Comb based solutions such as gain-switched optical frequency combs (GS-OFCs $)^{[11]}$ and mode locked lasers (MLLs) ${ }^{[4]}$ can provide pairs of coherent carriers for heterodyning but lack a high degree of tunability in operational wavelength and carrier frequency spacing, respectively. Integrated dual laser solutions providing higher levels of tunability have also been proposed using two distributed Bragg reflector (DBR) lasers $^{[12,13]}$ with Carpintero et al. ${ }^{[12]}$ showing flexible $\mathrm{THz}$ operation on a hybrid InP-polymer platform. The advantages of silicon photonics (SiP) are exploited by Hulme et al. ${ }^{[14]}$ who demonstrate mm-wave carrier generation from 1$112 \mathrm{GHz}$ with a wavelength tuning range of 42 $\mathrm{nm}$ using a dual external cavity laser (ECL) integrated SiP circuit.

In this work, a tunable $\operatorname{InP}-\mathrm{Si}_{3} \mathrm{~N}_{4}$ based hybrid integrated dual laser module is successfully deployed for the first time in a heterodyne A-RoF fronthaul link, incorporating both $10 \mathrm{~km}$ fiber and $60 \mathrm{GHz}$ wireless transmission over up to $2 \mathrm{~m}$. The dual laser device, which offers ultra wide wavelength tuning (up to $70 \mathrm{~nm}$ for each laser ${ }^{[15]}$ ) and relative carrier frequency differences incorporating $\mathrm{mm}$-wave and $\mathrm{THz}$ frequency ranges $^{[16]}$, is combined in the fronthaul system with a phase noise cancelling (PNC) receiver which eradicates the need for a receiver local oscillator (LO) ${ }^{[17]}$. The resultant system supports wavelength flexible $60 \mathrm{GHz}$ A-RoF delivery, with transmission of 5G 64-QAM orthogonal frequency division multiplexing (OFDM) exhibiting error vector magnitudes (EVM) as low 


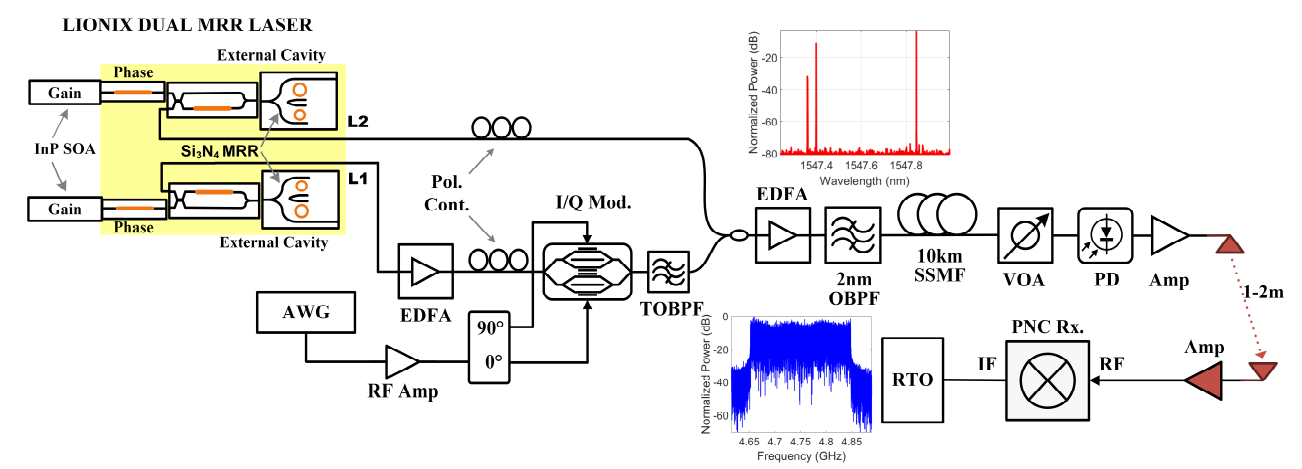

Fig. 1: Dual laser module schematic and experimental setup. Elements in red indicate components added to the system for wireless transmission. Insets show transmitted optical spectrum (red) and a received IF OFDM signal (blue).

as $5 \%$ over the full link.

\section{MRR based Dual Tunable Laser Module}

The $\operatorname{InP}-\mathrm{Si}_{3} \mathrm{~N}_{4}$ hybrid integrated dual laser module comprises two on-chip lasers, each with their own output fiber, and is graphically represented in Fig.1. Each laser consists of an independent InP semiconductor optical amplifier (SOA) hybrid coupled to $\mathrm{Si}_{3} \mathrm{~N}_{4}$ feedback circuit which incorporates two micro-ring resonators (MRRs) in a Vernier configuration. Both lasers have associated phase and Mach-Zehnder interferometer-based tunable coupler sections which, along with the MRRs, can be thermooptically controlled to vary the lasers' wavelength and output power. A detailed device description and characterization is given in our previous work [15], and shows laser tuning ranges of $70 \mathrm{~nm}$ each and high side mode suppression ratio (SMSR) of $>50 \mathrm{~dB}$. In this work, fine thermo-optic tuning of each lasers' associated sections was undertaken such that a relative carrier frequency difference of $56 \mathrm{GHz}$ was exhibited at four separate central wavelengths within the $\mathrm{C}$-band.

\section{Experimental Setup}

The heterodyne A-RoF experimental setup is shown in Fig. 1. The output from laser 1 (L1) is set to $-3 \mathrm{dBm}$ and is then amplified to overcome the losses introduced by the modulation arm of the transmitter. The output of laser 2 (L2) is set to $+1 \mathrm{dBm}$ and assigned as the unmodulated optical carrier. An electrical 64-QAM OFDM signal (800 data subcarriers, $195 \mathrm{MHz}$ bandwidth, $244 \mathrm{kHz}$ subcarrier spacing and data rate of $1.17 \mathrm{~Gb} / \mathrm{s}$ ) at an intermediate frequency (IF) of $4.75 \mathrm{GHz}$, was produced by the arbitrary waveform generator (AWG) operating at $20 \mathrm{GSa} / \mathrm{s}$. The signal was amplified and fed to a $90^{\circ}$ hybrid coupler and $\mathrm{I} / \mathrm{Q}$ Mach-Zehnder Modulator (MZM) biased close to the null point to perform optical single sideband (O-SSB) modulation on the optical carrier (L1). Complete suppression of the upper sideband was achieved using an optical band pass filter (OBPF) following modulation - considering the 20 $\mathrm{dB}$ modulator extinction. The modulated and the unmodulated optical carriers, which are $56 \mathrm{GHz}$ apart, are coupled using a 50:50 coupler and the combined output is amplified by the Erbium doped fiber amplifier (EDFA) to a total power of $+3 \mathrm{dBm}$ before it enters the $10 \mathrm{~km}$ fiber. A $2 \mathrm{~nm}$ OBPF is used for filtering out the out-of-band amplified spontaneous emission (ASE) noise. The two optical carriers beat together at the 70 $\mathrm{GHz}$ PIN photodetector and produce a mm-wave carrier at $56 \mathrm{GHz}$ and OFDM sideband at 60.75 $\mathrm{GHz}(56 \mathrm{GHz}+4.75 \mathrm{GHz})$. The composite $\mathrm{mm}$ wave signal (carrier and data signal are amplified and then transmitted over a wireless link of 1 - 2 $\mathrm{m}$ using a set of $20 \mathrm{~dB}$ gain directional horn antennae. The transmission of the $\mathrm{mm}$-wave data signal alongside a phase noise correlated carrier, enables phase noise and frequency offset cancellation using an analog electrical PNC receiver structure which is described in detail in ${ }^{[5}$, 17]. Mixing of the mm-wave carrier and data sideband terms at the PNC stage produces a clean IF OFDM signal which is then captured by a real time oscilloscope (RTO) at $50 \mathrm{GSa} / \mathrm{s}$ before offline processing consisting of synchronization, channel estimation and equalisation and EVM measurement was performed.

\section{Results and Discussion}

In order to assess the suitability of the photonic integrated dual laser source for $\mathrm{mm}$-wave distribution in a flexible network, the performance of the outlined mm-wave A-RoF system is analyzed using four different sets of wavelengths (attained through appropriate biasing of the device's sections) across the C-band.. Four different transmission scenarios are then evaluated for each wavelength pair while maintaining a constant relative carrier difference of $56 \mathrm{GHz}$. Fig. 2(i) shows normalized optical spectra for these four sets of wavelengths along with a modulated $5 G$ NR compatible OFDM data signal. The four insets in Fig. 2(i) show the corresponding 64-QAM constellations of the demodulated $60.75 \mathrm{GHz} \mathrm{mm}$-wave signal at these wavelengths after optical back-to-back 


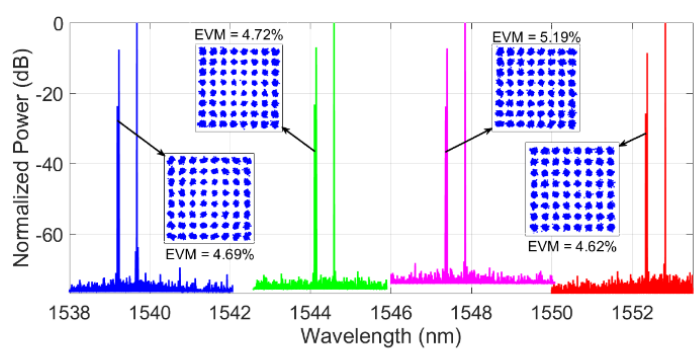

(i)

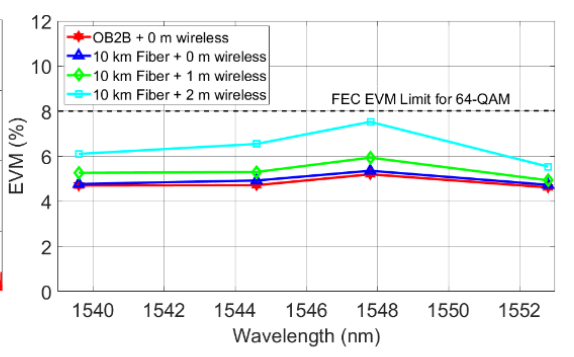

(ii)

Fig. 2: (i) Superimposed spectra of wavelengths after $2 \mathrm{~nm}$ OBPF with respective constellations of the demodulated 60.75 $\mathrm{GHz} \mathrm{mm}$-wave signal and EVM values, (ii) EVM performance of the received signal with respect to the wavelength.

(OB2B) transmission - which does not include wireless transmission. The excellent performances across the $\mathrm{C}$-band $\mid$ (which are also shown by the red curve in Fig 2(ii)) of between 4.6 and $5.2 \%$ EVM highlight the wavelength tuning capabilities of the device, as well as the ability of the PNC receiver to mitigate the effects of phase noise (PN) and frequency offset which can arise from the independent nature of the lasers' on-chip gain sections. A small degradation in the performance at the $1547.8 \mathrm{~nm}$ wavelength pair is attributed to a reduction in the combined EDFA gain response at that wavelength, as evidenced by the corresponding normalized spectrum (pink) in Fig. 2(i). Consequently, a slightly elevated EVM value is obtained for all transmission results obtained at this wavelength (see Fig. 2(ii)). Fig. 2(ii) also shows that the addition of transmission over $10 \mathrm{~km}$ of fiber has a negligible effect on system performance compared to the OB2B case for all four wavelength pairs - the received optical power (ROP) is set at $-2 \mathrm{dBm}$ in these cases.

Wireless mm-wave transmission is experimentally undertaken by adding two horn antennae each with a pass band of $55-65 \mathrm{GHz}$ and an additional receiver side electrical amplifier required to boost the input power to the PNC receiver. The line of sight wireless link was manually adjusted to maximise the received power for transmission distances of 1 and 2 m's. Fig. 2 (ii) shows that the addition of a $1 \mathrm{~m}$ wireless link following $10 \mathrm{~km}$ fronthaul transmission (green curve) results in a small $(\sim 0.5 \%)$ degradation in EVM across all tested wavelength pairs. This is attributed to the reduced electrical signal-to-noise ratio (SNR) at the receiver due to signal attenuation over the wireless link. Nevertheless, excellent performance below $6 \%$ was achieved in all cases over the full fiber-wireless link.

Increasing the wireless distance to $2 \mathrm{~m}$ results in a further $1-1.5 \%$ degradation in EVM across the range, and this is due to the combined effects of marginally elevated PD nonlinearity (as the ROP in this case was increased to $0.5 \mathrm{dBm}$ in order to obtain a reasonable signal level at the PNC receiver), as well as additional signal attenuation over the increased wireless link distance. Again, good performance, below the forward error correction (FEC) limit, of between 6 and $7.8 \%$ EVM can be observed for the received $1.17 \mathrm{~Gb} / \mathrm{s}$ OFDM signal. It should be noted that a more advanced wireless mm-wave link design, such as beam-forming operation using a phase array antenna (PAA) for example, could greatly increase the achievable wireless transmission distance for the presented system ${ }^{[18]}$.

\section{Conclusion}

The experimental results presented in this work demonstrate the ability of a hybrid integrated InP$\mathrm{Si}_{3} \mathrm{~N}_{4}$ dual tunable laser module to provide flexible carrier assignment, across a range of wavelengths in the C-band, for remote $\mathrm{mm}$-wave generation. When implemented in tandem with an analog PNC receiver circuit, excellent performances of $\sim 5 \%$ EVM are achieved on all test wavelengths over the full fibre fronthaul link and a $1 \mathrm{~m}$ wireless distance, with negligible degradation compared to the back-to-back cases. The results demonstrate, for the first time, how an integrated dual MRR-based laser module can provide a highly re-configurable siliconbased platform for the successful delivery of $5 \mathrm{G}$ A-RoF mm-wave services - in this case delivering a raw mobile data rate of $1.17 \mathrm{~Gb} / \mathrm{s}$ at $60.75 \mathrm{GHz}$.

Compared to InP-based solutions, the used silicon nitride approach provides the potential for smaller form-factor, greater cost efficiency and a higher yield device fabrication, as well as ease of integration with surrounding optical and electronic components; ultimately paving the way for a fully integrated optical $/ \mathrm{mm}$-wave transmitter.

Overall, the work shows that wide tunability afforded by the integrated dual laser module in combination with advanced system design is a highly promising approach enabling photonic mm-wave networking over an optical access infrastructure. This holds particular relevance for future converged optical networks exploiting flexibility in the wavelength domain.

\section{Acknowledgements}

The authors would like to acknowledge Dr. Eamonn Martin for useful discussions. This work has emanated from research supported by SFI under grant numbers 18/SIRG/5579, 13/RC/2077_P2, \& 12/RC/2276_P2. 


\section{References}

[1] H. Tataria, M. Shafi, A. F. Molisch, M. Dohler, H. Sjöland and F. Tufvesson, "6G Wireless Systems: Vision, Requirements, Challenges, Insights, and Opportunities," in Proceedings of the IEEE,

[2] Christina Lim and Ampalavanapillai Nirmalathas, "Radio-Over-Fiber Technology: Present and Future," J. Lightwave Technol. 39, 881-888 (2021)

[3] P. T. Dat, A. Kanno, N. Yamamoto and T. Kawanishi, "Seamless Convergence of Fiber and Wireless Systems for 5G and Beyond Networks," in Journal of Lightwave Technology, vol. 37, no. 2, pp. 592-605, 15 Jan.15, 2019

[4] A. Delmade et al., "Optical Heterodyne Analog RadioOver-Fiber Link for Millimeter-Wave Wireless Systems," in Journal of Lightwave Technology, vol. 39, no. 2, pp. 465-474, 15 Jan.15, 2021,

[5] D. Dass, S. O'Duill, A. Delmade, and C. Browning, "Analysis of Phase Noise in a Hybrid Photonic/Millimetre-Wave System for Single and MultiCarrier Radio Applications," Appl. Sci., vol. 10, no. 17, Art. no. 17, Jan. 2020,

[6] S. Noor, P. Assimakopoulos and N. J. Gomes, "A Flexible Subcarrier Multiplexing System With Analog Transport and Digital Processing for 5G (and Beyond) Fronthaul," in Journal of Lightwave Technology, vol. 37, no. 14, pp. 3689-3700, 15 July15, 2019

[7] Jim (Shihuan) Zou, Silviu Adrian Sasu, Mirko Lawin, Annika Dochhan, Jörg-Peter Elbers, and Michael Eiselt, "Advanced optical access technologies for nextgeneration (5G) mobile networks [Invited]," J. Opt. Commun. Netw. 12, D86-D98 (2020)

[8] P. Iovanna et al., "Future proof optical network infrastructure for 5G transport," in IEEE/OSA Journal of Optical Communications and Networking, vol. 8, no. 12, pp. B80-B92, December 2016

[9] Colm Browning, Qixiang Cheng, Nathan C. Abrams, Marco Ruffini, Liang Yuan Dai, Liam P. Barry, and Keren Bergman, "A Silicon Photonic Switching Platform for Flexible Converged Centralized-Radio Access Networking," J. Lightwave Technol. 38, 5386-5392 (2020)

[10] X. Guan, R. Dubé-Demers, W. Shi and L. A. Rusch, "Heterogeneous Optical Access Networks: Enabling Low-Latency $5 \mathrm{G}$ Services With a Silicon Photonic Smart Edge," in Journal of Lightwave Technology, vol. 39, no. 8, pp. 2348-2357, 15 April15, 2021.

[11] C. Browning et al., "Gain-Switched Optical Frequency Combs for Future Mobile Radio-Over-Fiber MillimeterWave Systems," in Journal of Lightwave Technology, vol. 36, no. 19, pp. 4602-4610, Oct.1, 2018,

[12] Carpintero, G., Hisatake, S., de Felipe, D. et al. Wireless Data Transmission at Terahertz Carrier Waves Generated from a Hybrid InP-Polymer Dual Tunable DBR Laser Photonic Integrated Circuit. Sci Rep 8, 3018 (2018).

[13] Y. Liu et al., "Dual-wavelength DBR laser integrated with high-speed EAM for THz communications," Opt. Express, vol. 28, no. 7, p. 10542, Mar. 2020.

[14] Jared Hulme, MJ Kennedy, Rui-Lin Chao, Linjun Liang, Tin Komljenovic, Jin-Wei Shi, Bogdan Szafraniec, Doug Baney, and J. E. Bowers, "Fully integrated microwave frequency synthesizer on heterogeneous silicon-III/V," Opt. Express 25, 2422-2431 (2017)

[15] Devika Dass, Marcos Troncoso Costas, Liam P. Barry, Sean O'Duill, Chris G. H. Roeloffzen, Douwe Geuzebroek, Guillermo Carpintero, Robinson Cruzoe Guzman, and Colm Browning, "28 GBd PAM-8 transmission over a $100 \mathrm{~nm}$ range using an $\operatorname{InP}-\mathrm{Si}_{3} \mathrm{~N}_{4}$ based integrated dual tunable laser module," Opt. Express 29, 16563-16571 (2021)

[16] R. Guzman et al., "Widely Tunable RF Signal Generation Using an InP/Si3N4 Hybrid Integrated DualWavelength Optical Heterodyne Source," in Journal of Lightwave Technology

[17] C. Browning, A. Delmade, Y. Lin, J. Poette, H. H. Elwan and L. P. Barry, "Phase noise robust optical heterodyne system for reduced complexity millimeter-wave analog radio-over-fibre," 45th European Conference on Optical Communication (ECOC 2019), 2019, pp. 1-4

[18] I. F. Akyildiz, C. Han and S. Nie, "Combating the Distance Problem in the Millimeter Wave and Terahertz Frequency Bands," in IEEE Communications Magazine, vol. 56, no. 6, pp. 102-108, June 2018 